Author Accepted Version. Final version published as: Temple, J. B., Brijnath, B., Enticott, J., Utomo, A., Williams, R., \& Kelaher, M. (2020). Discrimination reported by older adults living with mental health conditions: types, contexts and association with healthcare barriers. Social Psychiatry and Psychiatric Epidemiology, 1-12.

Discrimination reported by older adults living with mental health conditions: types, contexts and association with healthcare barriers

\author{
Jeromey B. Temple, Ph.D \\ Associate Professor and Head, \\ Demography and Ageing Unit \\ Melbourne School of Population and Global Health \\ University of Melbourne \\ jeromey.temple@unimelb.edu.au \\ Bianca Brijnath, Ph.D \\ Associate Professor and Director, Social Gerontology \\ National Ageing Research Institute (NARI) \\ b.brijnath@nari.edu.au \\ Joanne Enticott, Ph.D \\ Monash Centre for Health Research and Implementation, Monash University \\ Southern Synergy, Department of Psychiatry, Monash University \\ Joanne.Enticott@monash.edu \\ Ariane Utomo, Ph.D \\ School of Geography \\ Faculty of Science \\ University of Melbourne \\ ariane.utomo@unimelb.edu.au \\ Ruth Williams, Ph.D \\ Demography and Ageing Unit \\ Melbourne School of Population and Global Health \\ University of Melbourne \\ ruth.williams@unimelb.edu.au \\ Margaret Kelaher, Ph.D \\ Professor \\ Centre for Health Policy \\ Melbourne School of Population and Global Health \\ University of Melbourne \\ mkelaher@unimelb.edu.au
}




\title{
Discrimination reported by older adults living with mental health conditions: Types, contexts and association with healthcare barriers
}

\begin{abstract}
\end{abstract}
Objective: Australian policy-making needs better information on the prevalence, context and types of discrimination reported by people living with mental health conditions and the association of exposure to discrimination with experiencing a barrier to accessing healthcare. Methods: Secondary data analysis using the national representative General Social Survey 2014 to examine discrimination and healthcare barriers. Multivariable logistic regression was used to examine the association between discrimination and barriers to healthcare.

Results: Around $10 \%$ of older adults without mental health conditions reported an instance of discrimination in the last 12 months, compared to $22-25 \%$ of those with mental health conditions. Approximately $20 \%$ with mental health conditions attributed discrimination to their health conditions, along with other characteristics including age. Discrimination was reported in settings important to human capital (e.g., healthcare, workplace), but also in general social and public contexts. Everyday discrimination $(\mathrm{OR}=2.11 \mathrm{p}<.001)$, discrimination in healthcare $(\mathrm{OR}=2.92 \mathrm{p}<.001)$, and discrimination attributed to the person's health condition $(\mathrm{OR}=1.99$ $p<.05)$ increased the odds of experiencing a barrier to care two-to-three-fold. For each type of discrimination reported (e.g., racism, ageism etc.), the odds of experiencing a barrier to care increased 1.3 times $(\mathrm{OR}=1.29 \mathrm{p}<.01)$.

Conclusion: This new population-level evidence shows older adults with mental health conditions are experiencing discrimination at more than two-fold compared to those without mental health conditions. Discrimination was associated with preventing or delaying healthcare access. These findings indicate that future strategies to promote mental healthcare in underserved groups of older people will need to be multidimensional and consideration given to address discrimination.

\section{Keywords:}

Ageing, Ageism, Mental health, Discrimination, Healthcare seeking, Healthcare barriers. 


\section{Discrimination reported by older adults living with mental health conditions: Types, contexts and association with healthcare barriers}

\section{Introduction}

A widely recognised barrier to achieving social and economic participation for people living with mental health conditions is stigma and discrimination [1]. Stigma and discrimination have a profound effect on the lives of people with mental health conditions, magnifying their suffering, hindering treatment and recovery, and altering their life course [2]. Stigma and discrimination affect not only individuals living with mental health conditions, but also those around them. It is, therefore, unsurprising that stigma has been recognised as a topic requiring urgent research and has been identified as one of the grand challenges in mental health research [3].

Stigma was originally defined by Goffman [4] as a deeply discrediting attribute (e.g. age, physical abnormality, failure of character, race, religion, nationality) assigned to an individual, which a larger social group then uses as the basis to discredit and exclude. Extending this definition, Link and Phelan [5] defined stigma as the co-occurrence of labelling, stereotyping, separation, status loss, and discrimination in a context in which power is exercised. Discrimination, seen as distinct from stigma, is a set of actions by which the stigmatised individual's life opportunities are reduced often through institutional forces that marginalise the individual [5,6]. A further focus of Link and Phelan's [7] is the subjective expectation and experience of being labelled and discriminated against, which in turn affects individuals in their daily lives.

However, these definitions are far from settled and debate continues around the theoretical scope of stigma and discrimination [8-10]. Indeed, some researchers advocate a preference for 'discrimination' over 'stigma' to highlight that the opportunities gained or denied through institutional access are the preferred target for change [11]. Others distinguish between public 
and self-stigma, whereby perceived or received discrimination is internalised, manifesting as shame, secrecy and a reluctance to disclose symptoms to others [12]. Conceptual ambiguities notwithstanding, for the purposes of this article, we utilise Link and Phelan's [5] conceptualisation of perceived stigma to investigate the subjective experiences of discrimination reported by older people with mental health conditions in Australia.

We focus our inquiry on people aged 55 years and over. In doing so, we seek to address two gaps in the current evidence: first, though there is considerable scholarship showing that perceived and/or received mental-health related stigma and discrimination in the broader population is associated with delay and/or avoidance in seeking help from mental health professionals [13,14], little is known about the types of discrimination reported by older people with mental health conditions. Yet, underutilisation of mental healthcare services among the older population with mental health conditions is common $[15,16]$. There is a link between underutilisation of mental healthcare services and other forms of physical health and medical services which contribute to adverse morbidity outcomes and premature mortality $[17,18]$.

Second, there is strong evidence that the stigma and discrimination associated with mental health conditions also intersect with other prejudices such as ageism and sexism [11]. Therefore, any discussion of stigma and discrimination must examine their inherently multidimensional nature in order to more fully understand the impact on those living with mental health conditions. Older adults living with mental health conditions may be at risk of enduring the double stigma arising from both their age and their mental health status [19-21]. Defined as pervasive misconceptions, negative attitudes and assumptions about older people - often as frail, anachronistic, burdensome, and dependent - ageism is ubiquitous in Western societies [22]. It is a barrier to effective public policy [23] as well as the delivery of quality healthcare, including screening, prevention, treatment and management of disease [24]. Healthcare providers are also more likely to hold more negative implicit attitudes towards older adults than the general population [25]. However, the extent to which ageism and mental 
health stigma and discrimination combine to affect health seeking is unclear. Using nationally representative cross-sectional data, our aim was to produce high quality population-level evidence to answer three questions regarding the experiences of discrimination reported by older Australians living with mental health conditions: (1) What is the prevalence of older Australians with mental health conditions reporting any form of discrimination? (2) Does the type (e.g., ageism) or context (e.g., healthcare) of discrimination differ by presence of a mental health condition? (3) Is exposure to discrimination associated with experiencing a barrier to healthcare?

\section{Methods}

Data

This paper is based on secondary data analysis from the 2014 General Social Survey (GSS) conducted by the Australian Bureau of Statistics (ABS) between March and June 2014 [26]. The GSS was first conducted in 2002, with subsequent surveys conducted in 2006, 2010, and 2014. Using face-to-face interviews along with prompt cards, the ABS collected information using a Computer Assisted Interviewing (CAI) questionnaire on a range of domains to understand the "multi-dimensional nature of relative advantage and disadvantage across the population, and to facilitate reporting on and monitoring of people's opportunities to participate fully in society" [26].

Designed to provide reliable national and State/Territory-level estimates, the survey was conducted across urban and rural areas of Australia. However, individuals living in very remote locations, and in discrete Aboriginal and Torres Strait Islander communities were excluded from the survey [26]. Information collected in the GSS included: health, family relationships, social and community involvement, education, employment, income and financial stress, assets and liabilities, housing and mobility, crime and safety, transport, attendance at culture and leisure venues, and sports attendance and participation [27]. 
The GSS included an initial sample of 18,574 private dwellings, of which 16,145 dwellings were used due to issues of scope or uninhabited dwellings. In total, $80 \%$ fully responded, yielding a sample of 12,932 people aged 15 years and over. From this final sample, we only included respondents aged 55 years and over, yielding a final sample size of 4,967 respondents. As noted elsewhere, the age group 55-59 presents a unique turning point in disability prevalence in Australia because from this age-group onwards, the age-specific prevalence of disability exceeds the population average [28]. It is common for other Australian studies on ageing to use 55 as a cut-off point given that many Australians can start accessing their private superannuation from the age of $55[29,30]$.

Data for the GSS were collected by the ABS under the provisions of the Census and Statistics Act (CSA) 1905. Confidential data and access to the Remote Access Data Laboratory (RADL) were made available to the authors for this study through the ABS and Universities Australia agreement. Ethics approval for this project was granted by the Melbourne School of Population and Global Health Human Ethics Advisory Group (HEAG) - Ethics ID: 1953686.1.

\section{Measures}

\section{Discrimination}

Following extensive testing and consultation with various data users and stakeholders, a module on experiences on discrimination was added to the GSS in 2014 [27]. Members of the reference group included representatives of State and Territory Government department and agencies, universities and social research organisations. Cognitive interviews in the form of semi-structured interviews were conducted in March and April 2013. The purpose of the cognitive interviews was to ascertain that new additions to the GSS, including the discrimination module, were understood by respondents. Following the cognitive interviews, field testing was conducted of the survey questionnaire and procedures from September to October 2013. 
Measurement of discrimination is operationalised as follows: "In the past 12 months, that is since this time last year, do you feel that you have experienced discrimination or have been treated unfairly by others?" Those responding yes, were further prompted: "In the past 12 months, in which places or situations do you feel that you have experienced discrimination or have been treated unfairly?" A prompt card was then shown to the respondent listing: "at home, at work, at the shop, on public transport, at school or university, in a restaurant or bar, on the street or in a public place, online, applying for work/jobs, applying for or keeping a flat/apartment or housing of any kind, dealing with police, dealing with the courts, dealing with government officials, dealing with people involved in healthcare, other or don't know".

The characteristic attributed to discrimination was measured by asking "Thinking about your most recent experience of discrimination in Australia, do you think it was because of any of the following?" A prompt card was then displayed (Prompt Card F27) listing: "your skin colour, your nationality, race or ethnic group, the language you speak, the way you dress or your appearance, your gender, your age, a disability or health issue, your marital status, your family status, your sexual orientation, your occupation, your religious beliefs, your political position or other".

\section{Mental Health Conditions}

Mental health conditions were collected in two components of the survey instrument, the longterm conditions module (module 6.2) and disability module (6.3). For long-term health conditions, respondents were asked "Thinking of conditions lasting six months or more, have you ever been told by a doctor or nurse that you have any of these conditions?" A prompt card was then shown to the respondent (Prompt card D1). Among the list included depression or feeling depressed, behavioral or emotional disorders, dependence on drugs or alcohol, feeling anxious or nervous, and problems learning or understanding things. The ABS defines a longterm mental health condition as the presence of any of these conditions. 
People with a disability in the GSS are defined as those who identify "a restriction to their core activities, a schooling or employment restriction as well as those without a specific restriction" [26]. Conditions defined as a mental health disability included intellectual, psychological or head injury, stroke or brain damage. By definition, all people with a mental health disability have a mental health condition.

\section{Barriers to healthcare}

Measurement of barriers to health care was collected in the self-perceptions of health and healthcare delays module (module 6.1). Respondents were asked "In the last 12 months, was there ever a time that you needed healthcare but could not get it?" Those who responded yes to this question were coded as experiencing a healthcare barrier. The ABS has utilized this measure across numerous surveys including the National Health Survey, Survey of Disability, Ageing and Carers and the Patient Experiences Survey. National and international population level studies aiming to estimate the prevalence of unmet needs for healthcare have also used a similar instrument of self-reported/subjective barriers to health care, drawn from a yes/no question in national and cross-country surveys phrased along the line of: "Was there a time in the last 12 months when you needed health care but did not receive it?" [31-33].

\section{Measurement of financial wellbeing}

To examine the association between discrimination and barriers to healthcare, it is important to control for predisposing, need, and enabling factors providing access [34]. One important measure of enabling factors is household financial wellbeing. The GSS also included a number of detailed items measuring incidence of cash flow problems and dissaving behaviors. Cash flow problems recorded in the GSS included difficulties paying (i) electricity, gas or telephone bills (ii) mortgage or rent payments, (iii) car registration or insurance, and (iv) minimum credit card payments. It also included the prevalence of selling belongings to pay for bills, going without meals, being unable to heat their home, and seeking financial support from friends, family, or from welfare and community organisations. In addition to cash flow problems, the 
GSS also recorded a number of measures of dissaving behaviors. That is, drawing down on assets or taking out liabilities to meet current living costs. Among the asset dissaving measures were drawing on accumulated savings or term deposits, selling household goods or jewelry, and selling shares, stocks or bonds or other assets. The liability dissaving measures included reducing home loan repayments, increasing the balance on credit cards by $\$ 1,000$ or more, entering into a loan agreement with family or friends or taking out a personal loan. The GSS questionnaire was administered in a way to ensure that these measures were undertaken specifically to meet day-to-day living expenses. As an indicator of financial wellbeing, the counts of the number of cash flow and dissaving behaviours was calculated and included as an important control variable.

\section{Statistical tests}

For the bivariate analyses, tests of the weighted prevalence of discrimination, its types and contexts stratified by mental health conditions and disabilities are presented. To examine the association between exposure to discrimination and experiencing a barrier to healthcare, multivariable logistic regression models were fitted. A range of control variables were tested in the modelling including age, sex, marital status, gross equivalent household income in deciles, country of birth, education, income source, and health conditions. To maintain the most parsimonious specification, the final models retained all variables significant at the $95 \%$ critical value, including:

- $\quad$ Age $(55-64,65-74,75+)$.

- Region of residence, classified according the ABS (major cities, inner regional areas, other areas).

- Financial wellbeing (measured by the number of financial stressors reported in the previous 12 months as outlined above).

- Health conditions (measured by the number of long-term health conditions reported by the respondent). 
- Measures of discrimination.

Although many covariates may be associated with experiencing a barrier to health care, we present relatively parsimonious models with controls for selected predisposing, need and enabling factors as outlined above. Nonetheless, our model specification is robust to multicollinearity and is confirmed by standard model selection techniques. As will be shown, our multivariable results are highly consistent with the underlying bivariate relationships.

Two groups of models are presented. The first group of analyses considered all people aged 55 years and over. In these analyses, the association between discrimination and barriers to healthcare was examined through everyday discrimination, i.e., any form of discrimination (model $A$; outcome variable=1 if respondent said yes to experiencing any form of discrimination), discrimination in healthcare (model B; outcome variable $=1$ if respondent said yes to experiencing discrimination when 'dealing with people involved in healthcare') and discrimination due to health (model $\mathrm{C}$; outcome variable=1 if respondent said yes that the discrimination was attributed to disability or health condition). A categorical variable was formed indicating the unique intersection of the presence of each form of discrimination with mental health conditions.

The second group of analyses considered those aged 55 years and over with mental health conditions. In these analyses, the association between discrimination and barriers to healthcare was examined through everyday discrimination (model $D$; outcome variable=1 if respondent said yes to experiencing any form of discrimination), discrimination in healthcare (model E; outcome variable=1 if respondent said yes to experiencing discrimination when 'dealing with people involved in healthcare'), discrimination due to health (model F; outcome variable $=1$ if respondent said yes that the discrimination was attributed to disability or health condition) and counts of the number of discrimination types reported (model G). 


\section{Results}

Before turning to the key findings from this study, it is important to note some key demographic characteristics of the GSS. In the full GSS sample, ABS analyses show demographic and health estimates are highly comparable to other ABS surveys [35]. For example, the 2011-12 National Health Survey estimates $5.3 \%$ of the population live with a psychological disability, compared with $5.9 \%$ in the GSS. Approximately $64 \%$ of people are employed in the GSS, compared with $63 \%$ of people in the 2014 Labour Force Survey. Between $13-14 \%$ of people in both the GSS and 2009-10 Household Expenditure Survey could not raise $\$ 2000$ within a week. Finally, about $30 \%$ of respondents to both the GSS and 2011-12 Survey of Income and Housing report 'renter' as their household tenure status. Across these and a variety of demographic and economic measures, the GSS is highly consistent with other nationally representative surveys. In the GSS sample of Australians aged 55 and over, $23 \%$ of respondents were aged 75 or over, about three quarters of respondents rated their health as 'good', 'very good' or 'excellent' and approximately $67 \%$ were born in Australia as were those living in major Australian cities.

Table 1 displays the prevalence of any form of discrimination disaggregated by presence and type of a mental health condition. Around $10 \%$ of older Australians without mental health conditions reported an instance of discrimination in the last 12 months, compared with $22 \%$ $25 \%$ of those with a mental health condition. Among those with long-term mental health conditions, prevalence is elevated for those reporting a behavioural or emotional disorder (37\%), however, this group only accounts for approximately $10 \%$ of this group. Prevalence of discrimination is lower for those reporting dependences on drugs or alcohol $(11.9 \%)$ and is comparable to the population without mental health conditions, but again this group is relatively small in the older population. Among those with a mental health disability, just under one third of those with a psychological $(28.1 \%)$ or head injury, stroke or brain damage $(26.6 \%)$ reported discrimination. 


\section{[TABLE 1]}

Noting the significantly higher prevalence of discrimination reported by older people with mental health conditions, Table 2 presents the distribution of types of discrimination, classified by mental health status. That is, among people reporting discrimination, the reasons that respondents attributed to experiencing it are shown. Unsurprisingly, relative to those without a long-term mental health condition, those with a mental health condition were considerably more likely to attribute discrimination to a disability or health condition (10.4\% versus $20 \%)$. This is important, as it indicates that other factors explain the experience of discrimination among older people with mental health conditions. Those with mental health conditions were also more likely to cite marital or family status and marginally more likely to cite the language they speak (9.4\% versus 5.1\%). These results are broadly replicated for those with mental health disabilities, although almost one in three cite a disability of health condition as a reason for discrimination. Moreover, around $12 \%$ also cited 'the way you dress or your appearance'. Across all groups, age is cited by approximately one third of those as a reason for discrimination.

\section{[TABLE 2]}

In addition to differing in prevalence and reasons, the contexts for discrimination differed for older people with and without mental health conditions. (Table 3). Approximately one quarter of those with mental health conditions cited healthcare as the context of discrimination, relative to around $10 \%$ of those without mental health conditions. Approximately one in three with a mental health condition report on the street or in a public place, relative to two in five of those without a mental health condition. For those with a mental health disability, approximately $28 \%$ reported dealing with government officials as the source of discrimination, compared with $14 \%$ of those without mental health conditions. Although workplace discrimination was higher 
for those without mental health conditions, almost one in three respondents with a mental health condition reported exposure in this context. Overall, however, these results indicate a broad range of contexts and situations in which older people in general, as well as those with mental health conditions, experience discrimination.

\section{[TABLE 3]}

Our final research aim was to examine the association between exposure to discrimination and the likelihood of reporting a barrier to healthcare. Table 4 presents odds ratios from logistic regression models of experiencing a barrier to healthcare in the full population aged 55 years and over. With controls included, presence of long-term health conditions and discrimination was significantly associated with an increased odds of experiencing a barrier to care. Compared to those with no mental health conditions and no exposure to discrimination, those with mental health conditions were about 1.7 times more likely to experience a barrier to healthcare (Everyday discrimination $[A] \mathrm{OR}=1.67 \mathrm{p}<.01$, discrimination in healthcare $[\mathrm{B}]$ OR=1.74 $p<.01$, discrimination due to health $[C]$ OR $=1.72 p<.01$ ). Experiencing discrimination, but without the presence of mental health conditions, was associated with a 2.5 to 5.5 increase in odds of experiencing a barrier to healthcare (Everyday discrimination $[A]$ $\mathrm{OR}=2.47 \mathrm{p}<.001$, discrimination in healthcare $[\mathrm{B}] \mathrm{OR}=5.57 \mathrm{p}<.001$, discrimination due to health $[C]$ OR $=2.7 p<.05)$. For those with mental health conditions, experiencing discrimination was associated with a 3.6 to 5.1 fold increase in the odds of experiencing a barrier to healthcare (Everyday discrimination [A] $O R=3.55 p<.001$, discrimination in healthcare $[B]$ OR=5.1 $p<.001$, discrimination due to health $[C]$ OR $=3.6 p<.001)$. Postestimation tests of the underlying logic coefficients indicate that the strength of the association between exposure to discrimination and healthcare barriers is similar between those with and without mental health conditions (chi $=1.82 p<.1[A]$ chi $=0.03 p<.1[B]$ chi $=0.23 p<.1[C]$ ). 
Although not directly central to our research question, results in table 4 also show important associations between the control variables and experiencing a barrier to health care - with results robust across all models (A, B and C). Relative to the youngest age group (55-64), those aged $65-74([A]$ OR=0.66 $p<.05[B]$ OR=0.61 $p<.01[C]$ OR=0.61 $p<.01)$ or 75 and over $([A]$ OR $=0.54 p<.01[B]$ OR=0.48 $p<.001[C]$ OR=0.48 $p<.001)$, were less likely to cite a barrier to health care. People living in outer regional or remote areas of Australia were more likely to experience a barrier to care relative to those living in major cities $([A]$ OR=1.74 $p<.001[B]$ $\mathrm{OR}=1.79 \mathrm{p}<.001[\mathrm{C}] \mathrm{OR}=1.80 \mathrm{p}<.001$ ). However, there was no difference in experiencing a barrier to care between major cities and inner regional areas of Australia ( $p>.1)$. Across all models, increasing levels of financial stress and presence of multiple long-term health conditions was strongly associated with experiencing a barrier to care $(p<.001)$.

\section{[TABLE 4]}

Table 5 limits the sample population to all older people with mental health conditions. Across each model (model $1-4$ ), discrimination was significantly associated with experiencing a barrier to care. Everyday discrimination $(\mathrm{OR}=2.11 \mathrm{p}<.001)$, discrimination in healthcare $(O R=2.92 p<.001)$ and discrimination attributed to the person's health condition $(O R=1.99$ $p<.05)$ all increased the odds of experiencing a barrier to care by two to three times. For each type of discrimination reported (e.g., racism, ageism, etc.), the odds of experiencing a barrier to care increased 1.3 times $(\mathrm{OR}=1.29 \mathrm{p}<.01)$.

\section{[TABLE 5]}

\section{Discussion}

Prevalence of older people reporting any form of discrimination

This work addresses a gap regarding information on mental health problems in Australia by exploring an available national survey for the subjective experiences of discrimination among 
older people with mental health conditions, and for the first time providing population-level evidence on the prevalence of discrimination, its multidimensional nature, and impact on health-seeking. To date, there have been no nationally-representative studies examining these factors, with much of the literature focused on media representations of older people with mental health conditions $[21,36]$, qualitative research $[37,38,12]$, scoping reviews $[39,21]$, and the vulnerabilities of specific groups such as older ethnic communities [40] and older lesbian, gay, bisexual, transgender, and queer communities [41]. The survey results show $22 \%-25 \%$ of older people with mental health conditions reported an instance of discrimination in the last 12 months, which was more than twice the rate of older adults without mental illness at approximately $10 \%$.

Does the type of discrimination differ by presence of a mental health condition?

Types of discrimination were found to be different in older people with and without mental health conditions. Discrimination attributed to health conditions and age contributed more in those with mental health conditions. Approximately $20 \%$ of those with mental health conditions attributed discrimination to their health conditions, with a range of other characteristics, including age, as the reason for discrimination. Discrimination was reported in settings important to human capital (e.g., healthcare, workplace), but also in general social and public contexts. Although discrimination in the workplace context was lower among those with a mental health condition relative to those without, this finding simply reflects the lower labour force participation of older Australians living with mental ill health [42]. These findings accord with previous work on the multidimensional nature of stigma reported across the general population [43], but, for the first time, quantify the likelihood of experiencing discrimination among older Australians with mental health conditions. Older people are particularly affected by stigma surrounding mental health conditions, such as depression [43] and have poorer mental health literacy $[44,45]$. These factors frequently affect whether or when appropriate help is sought, whether it is adhered to, and how people respond to others with mental health conditions [39]. Discrepancies in experiences of stigma and discrimination 
may also be more of an issue for older people relative to younger people, because increasing acceptance over time within the community may be resulting in younger generations having more positive attitudes to mental health conditions [46]. However, research also shows that while social distance may be reduced for mental health conditions such as depression, stigmatising beliefs prevail about psychosis and schizophrenia $[43,47]$.

Discrimination occurred most commonly in healthcare contexts, when dealing with government officials and in general public spaces, which also match previous findings $[14,48,49]$. Normalising mental health symptoms as part of ageing rather than as treatable conditions, patronising older people, and reduced time during clinical consultations are well known discriminatory behaviours observed among health professionals in a range of settings $[50,51,12,52]$. Despite lower labour force participation among older people with health conditions, about one in three of those experiencing discrimination indicated the workplace as a key point of exposure to discrimination. These findings also correspond to other studies on the effects of discrimination related to ageism at work, underscoring how multiple factors such as age and presence of mental health conditions can amplify discrimination [53].

Is exposure to discrimination associated with experiencing a barrier to healthcare? The national survey showed that barriers to care declined with increasing age. This might be because individuals who are 65 years and older have access to the Commonwealth Home Support Programme (CHSP). Under the CHSP, older Australians are able to access services such as nursing care, allied health services, domestic assistance and personal care thereby offsetting some of the personal attributes that may make access to health care difficult. There is also a possibility of a survival selectivity in these cross sectional data. As wealthier individuals are more likely to have improved survival prospects relative to their financially disadvantaged peers; in the cross sectional GSS data, we are observing these individuals [54]. As shown in the modelling, lower levels of financial stress are shown to strongly mitigate against barriers to accessing health care, reinforcing the age-based results we observe. 
However, among those older people who did report discrimination, it was associated with delayed or no care irrespective of mental health condition status. The effects of discrimination for people with and without mental health conditions is to de-motivate healthcare access, and since older people with mental health conditions are more susceptible to discrimination, then they likely experience greater demotivation. For people with mental health conditions, everyday discrimination $(O R=2.11 p<0.001)$, discrimination in healthcare $(O R=2.92 p<0.001)$ and discrimination attributed to the person's health condition $(O R=1.99 p<0.05)$ all increased the odds of experiencing a barrier to care by two to three times. For each type of discrimination reported (e.g., racism, ageism, etc.), the odds of experiencing a barrier to care increased 1.3 times $(O R=1.29 p<0.01)$. This important finding illustrates that among the increasingly diverse population of older people with mental health conditions, the double stigma related to their mental health and age may be further compounded by other experiences of discrimination pertaining to race, sex, gender and lower socio-economic status - that have accumulated along their life course [55-57]. This finding also concords with previous work showing that access to mental healthcare diminishes by the relative socioeconomic status of areas, with the more disadvantaged areas four to five times less likely to receive key services than areas of least disadvantage [58]. Given that rural and remote Australia are more disadvantaged compared to metropolitan and regional Australia, this may explain why there is limited difference between cities and inner regional areas when compared to the more remote localities. Nevertheless, there are differences even within major cities by neighbourhood and socioeconomic status; it is just that in this national analysis that those differences may be partially flattened. In the general ageing and health scholarship, studies have demonstrated that addressing intersectionality of disadvantage is key to improve health disparities in later life [59-61].

The findings here resonate with previous research suggesting that there are important barriers to older people accessing mental health services [62]. The higher level of discrimination 
towards older people with mental health conditions tends to suggest that this is not due to seeing mental health conditions as a "normal" part of ageing [63] but rather due to the stigma and discrimination associated with these conditions. The results highlight the need to address discrimination against older people with mental health conditions in the health system and support the growing trend for the development specialist services to better meet the mental health needs of older people [64].

\section{Limitations and extensions}

In interpreting results from this study, it is important to consider the limitations of our analyses and data. Firstly, data from the GSS is cross-sectional. We cannot and do not draw causal relationships between discrimination and experiencing difficulties accessing healthcare. Nonetheless, our results indicate a strong association and is comparable to findings on discrimination and healthcare access and healthcare seeking more generally $[38,12]$. Second, our measures of discrimination are self-reported over a period of 12 months, which may result in underreporting of mental health conditions, especially among those with an undiagnosed mental health condition and recall bias for other respondents. Moreover, as a self-reported measure, these estimates of discrimination should be interpreted as 'perceived discrimination,' which may not fully correspond with actual levels of discrimination. Third, the GSS sampling frame was limited to people living in private dwellings, such as private family homes. Those living in high care nursing homes, hospitals or other non-private dwellings were not enumerated in the survey. Further data collections would be necessary to generalise the findings here to these populations. Related to this point, a further limitation of our analyses is the inability to consider the role of discrimination in describing barriers to health care facing older Aboriginal and Torres Strait Islander people living with mental health conditions. Unfortunately, the flag indicating Aboriginal status is not released in the GSS RADL microdata. Recent research underscores the considerable levels of racial discrimination encountered by older Aboriginal people and the deleterious consequences for their mental health $[65,66]$. Using the National Aboriginal and Torres Strait Islander Social Survey to examine the 
intersection of racism and barriers to health care among older Aboriginal people is a priority for the research team. Finally, the GSS has limited measures on specific mental health conditions in later life. For example, the prevalence of dementia is growing by virtue of population ageing and it would be useful to consider this group's exposure to discrimination and related association to healthcare barriers.

An area for further examination is the experience of discrimination in different sub-groups of the older population living with mental health conditions. We already know that prevalence of mental health conditions differ across Australia by demo-socio-economic factors and geography $[67,58,68]$. Further research is warranted to examine how reports of discrimination differ by demographic characteristics such as gender, country of birth, marital status and measures of socio-economic status. Such an analysis would provide important insights into the intersectionality of discrimination within the older population.

\section{Planning for future mental health care}

Taken together, these results underscore how stigma and setting are linked and alert us to the complex interventions needed to tackle this pervasive barrier to healthcare. Simply implementing a large-scale knowledge awareness campaign on the topic or seeking to empower older people to resist the stigma associated with mental health conditions is insufficient by itself. Rather, programs need to promote care engagement, mental health literacy, cultural competence, and family engagement alongside policy change to overcome the structural stigmas (e.g., ageism) that undermine policy agendas intended to promote mental healthcare. Understanding the diverse pathways through which these consequences may be realised may help identify targeted strategies to mitigate the harmful effects of stigma in particular settings, rather than adopting a universal one-size-fits-all approach. These new findings will inform future strategies to promote mental healthcare in older people and begin to address the health care utilisation in underserved groups. 


\section{Conclusion}

The nationally representative Australian GSS survey has produced high quality populationlevel evidence showing that: (1) older people with a mental health condition were approximately twice as likely to note an instance of discrimination than older people without a mental health condition; (2) instances of discrimination related to both their underlying health conditions and age; (3) exposure to discrimination often occurs in contexts critical to health and wellbeing (e.g., healthcare settings, in the workplace) and; (4) exposure to discrimination, regardless of the context or type, was associated with a two to three fold increase in the odds of experiencing a barrier to healthcare in Australia. These findings are new and are important to inform future interventions to promote access to mental health care in older people.

\section{Conflict of Interest}

On behalf of all authors, the corresponding author states that there is no conflict of interest.

\section{Description of Author Roles}

\section{Acknowledgement}

The Australian Bureau of Statistics (ABS) made data for this study available to the authors. The Australian Research Council's (ARC) Centre of Excellence in Population Ageing Research (CE1101029) funds author 1. The opinions expressed herein do not reflect those of the ABS or ARC. 


\section{References}

1. Hatzenbuehler ML, Phelan JC, Link BG (2013) Stigma as a fundamental cause of population health inequalities. American journal of public health 103 (5):813-821

2. Major B, O'brien LT (2005) The social psychology of stigma. Annu Rev Psychol $56: 393-421$

3. Collins PY, Patel V, Joestl SS, March D, Insel TR, Daar AS, Scientific Advisory B, the Executive Committee of the Grand Challenges on Global Mental H, Anderson W, Dhansay MA, Phillips A, Shurin S, Walport M, Ewart W, Savill SJ, Bordin IA, Costello EJ, Durkin M, Fairburn C, Glass RI, Hall W, Huang Y, Hyman SE, Jamison K, Kaaya S, Kapur S, Kleinman A, Ogunniyi A, Otero-Ojeda A, Poo M-M, Ravindranath V, Sahakian BJ, Saxena S, Singer PA, Stein DJ (2011) Grand challenges in global mental health. Nature 475 (7354):2730. doi:10.1038/475027a

4. Goffman E (1963) Stigma: notes on the management of spoiled identity. PrenticeHall, Englewood Cliffs, NJ

5. Link BG, Phelan JC (2001) Conceptualizing stigma. Annual review of Sociology 27 (1):363-385

6. Reidpath D, Brijnath B, Chan K (2005) An Asia Pacific six-country study on HIVrelated discrimination: Introduction. Aids Care 17 (sup2):117-127

7. Ritsher JB, Phelan JC (2004) Internalized stigma predicts erosion of morale among psychiatric outpatients. Psychiatry research 129 (3):257-265

8. Livingston JD, Boyd JE (2010) Correlates and consequences of internalized stigma for people living with mental illness: A systematic review and meta-analysis. Social science \& medicine $71(12): 2150-2161$

9. Rüsch N, Angermeyer MC, Corrigan PW (2005) Mental illness stigma: Concepts, consequences, and initiatives to reduce stigma. European psychiatry 20 (8):529-539

10. Thornicroft G, Rose D, Kassam A, Sartorius N (2007) Stigma: ignorance, prejudice or discrimination? The British Journal of Psychiatry 190 (3):192-193 
11. Pescosolido BA, Martin JK (2015) The stigma complex. Annual review of sociology $41: 87-116$

12. Polacsek M, Boardman GH, McCann TV (2019) Help-seeking experiences of older adults with a diagnosis of moderate depression. International journal of mental health nursing 28 (1):278-287

13. Clement S, Schauman O, Graham T, Maggioni F, Evans-Lacko S, Bezborodovs N, Morgan C, Rusch N, Brown JS, Thornicroft G (2015) What is the impact of mental healthrelated stigma on help-seeking? A systematic review of quantitative and qualitative studies. Psychological medicine 45 (1):11-27. doi:10.1017/s0033291714000129

14. Corrigan PW, Druss BG, Perlick DA (2014) The Impact of Mental Illness Stigma on Seeking and Participating in Mental Health Care. Psychological science in the public interest : a journal of the American Psychological Society 15 (2):37-70. doi:10.1177/1529100614531398

15. Byers AL, Arean PA, Yaffe K (2012) Low use of mental health services among older Americans with mood and anxiety disorders. Psychiatr Serv 63 (1):66-72. doi:10.1176/appi.ps.201100121

16. Crabb R, Hunsley J (2006) Utilization of mental health care services among older adults with depression. Journal of clinical psychology 62 (3):299-312. doi:10.1002/jclp.20231

17. Happell B, Scott D, Platania-Phung C (2012) Perceptions of barriers to physical health care for people with serious mental illness: a review of the international literature. Issues in Mental Health Nursing 33 (11):752-761

18. Harris C, Barraclough B (1998) Excess mortality of mental disorder. The British journal of psychiatry $173(1): 11-53$

19. de Mendonça Lima CA, Levav I, Jacobsson L, Rutz W (2003) Stigma and discrimination against older people with mental disorders in Europe. International Journal of Geriatric Psychiatry 18 (8):679-682

20. Graham N, Lindesay J, Katona C, Bertolote JM, Camus V, Copeland JR, de Mendonça Lima CA, Gaillard M, Gély Nargeot MC, Gray J (2003) Reducing stigma and 
discrimination against older people with mental disorders: a technical consensus statement. International Journal of Geriatric Psychiatry 18 (8):670-678

21. Thomas K, Shute R (2006) The old and mentally ill in Australia: Doubly stigmatised. Australian Psychologist 41 (3):186-192

22. Officer A, Schneiders ML, Wu D, Nash P, Thiyagarajan JA, Beard JR (2016) Valuing older people: time for a global campaign to combat ageism. Bulletin of the World Health Organization $94(10): 710$

23. Lloyd-Sherlock PG, Ebrahim S, McKee M, Prince MJ (2016) Institutional ageism in global health policy. BMJ 354:i4514

24. Dixon-Woods M, Cavers D, Agarwal S, Annandale E, Arthur A, Harvey J, Hsu R, Katbamna S, Olsen R, Smith L (2006) Conducting a critical interpretive synthesis of the literature on access to healthcare by vulnerable groups. BMC medical research methodology $6(1): 35$

25. Nash P, Stuart-Hamilton I, Mayer P (2014) The continuation of prejudice: addressing negative attitudes in nurse training and continuing professional education. Educational Gerontology 40 (1):53-60

26. Australian Bureau of Statistics (ABS) (2015) General social survey; summary results, 2014. Catalogue number 4159.0. Australian Bureau of Statistics, Canberra

27. Australian Bureau of Statistics (ABS) (2015) General Social Survey: User Guide, Australia, 2014 . Catalogue number 4159.0.55.002

28. Australian Bureau of Statistics (ABS) (2019) Disability, Ageing and Carers, Australia: Summary of Findings, 2018. Catalogue number 4430.0

29. Weiss C, Parkinson D, Duncan A (2015) Living Longer on Less:Women, Paid Work, and Superannuation in Victoria, Australia. SAGE Open 5 (3):2158244015597966. doi:10.1177/2158244015597966

30. Tan J, Ward L, Ziaian T (2010) Experiences of Chinese Immigrants and AngloAustralians Ageing in Australia:A Cross-cultural Perspective on Successful Ageing. Journal of Health Psychology 15 (5):697-706. doi:10.1177/1359105310368183 
31. Casey R (2015) Disability and unmet health care needs in Canada: a longitudinal analysis. Disability and health journal 8 (2):173-181

32. McColl MA, Jarzynowska A, Shortt S (2010) Unmet health care needs of people with disabilities: population level evidence. Disability \& Society 25 (2):205-218

33. Allin S, Masseria C (2009) Unmet need as an indicator of health care access. Eurohealth $15(3): 7$

34. Andersen RM (1995) Revisiting the Behavioral Model and Access to Medical Care: Does it Matter? Journal of Health and Social Behavior 36 (1):1-10. doi: $10.2307 / 2137284$

35. Australian Bureau of Statistics (ABS) (2015) Table 41590DO022 Data Comparability between GSS and other ABS sources. Canberra

36. Zhang $Y$, Jin $Y$, Stewart S, Porter J (2016) Framing responsibility for depression: How US news media attribute causal and problem-solving responsibilities when covering a major public health problem. Journal of Applied Communication Research 44 (2):118-135

37. Dawson S, Gerace A, Muir-Cochrane E, O'Kane D, Henderson J, Lawn S, Fuller $\mathrm{J}$ (2017) Carers' experiences of accessing and navigating mental health care for older people in a rural area in Australia. Aging Ment Health 21 (2):216-223. doi:10.1080/13607863.2015.1078283

38. Pettigrew S, Donovan R, Pescud M, Boldy D, Newton R (2010) Mature adults' attitudes to mental health service utilisation. Australian Psychologist 45 (2):141-150

39. Haralambous B, Lin, X., Dow, B., Jones, C., Tinney, J., Bryant, C. (2009) Depression in older age: a scoping study. National Ageing Research Institute, Melbourne 40. Johnstone MJ, Kanitsaki O (2008) Ethnic aged discrimination and disparities in health and social care: a question of social justice. Australasian Journal on Ageing 27 (3):110-115

41. Lyons A, Pitts M, Grierson J (2013) Factors related to positive mental health in a stigmatized minority: An investigation of older gay men. Journal of Aging and Health 25 (7):1159-1181 
42. Waghorn G, Lloyd C (2005) The employment of people with mental illness. Australian e-Journal for the Advancement of Mental Health 4 (2):129-171. doi:10.5172/jamh.4.2.129

43. Griffiths KM, Christensen H, Jorm AF (2008) Predictors of depression stigma. BMC psychiatry $8(1): 25$

44. Farrer L, Leach L, Griffiths KM, Christensen H, Jorm AF (2008) Age differences in mental health literacy. BMC Public Health 8 (1):125

45. Kim YS, Rhee TG, Lee HY, Park BH, Sharratt ML (2017) Mental health literacy as a mediator in use of mental health services among older korean adults. International psychogeriatrics 29 (2):269-279

46. Reavley NJ, Mackinnon AJ, Morgan AJ, Jorm AF (2014) Stigmatising attitudes towards people with mental disorders: A comparison of Australian health professionals with the general community. Australian \& New Zealand Journal of Psychiatry 48 (5):433-441

47. Reavley NJ, Jorm AF (2011) Stigmatizing attitudes towards people with mental disorders: findings from an Australian National Survey of Mental Health Literacy and Stigma. Australian \& New Zealand Journal of Psychiatry 45 (12):1086-1093

48. Kessler E-M, Agines S, Bowen CE (2015) Attitudes towards seeking mental health services among older adults: Personal and contextual correlates. Aging \& mental health $19(2): 182-191$

49. Mittal D, Sullivan G, Chekuri L, Allee E, Corrigan PW (2012) Empirical studies of self-stigma reduction strategies: a critical review of the literature. Psychiatric Services 63 (10):974-981

50. Allen RES, Wiles JL (2013) Receiving Support When Older: What Makes It OK? The Gerontologist 54 (4):670-682. doi:10.1093/geront/gnt047

51. Blancato RB, Ponder M (2015) The Public Policies We Need to Redress Ageism. Generations 39 (3):91-95 
52. Xiang X, Danilovich MK, Tomasino KN, Jordan N (2018) Depression prevalence and treatment among older home health services users in the United States. Archives of gerontology and geriatrics 75:151-157

53. Macdonald JL, Levy SR (2016) Ageism in the workplace: The role of psychosocial factors in predicting job satisfaction, commitment, and engagement. Journal of Social Issues $72(1): 169-190$

54. Disney R (1996) Can we afford to grow older?: a perspective on the economics of aging. Mit Press,

55. Conner KO, Copeland VC, Grote NK, Koeske G, Rosen D, Reynolds CF, 3rd, Brown C (2010) Mental health treatment seeking among older adults with depression: the impact of stigma and race. Am J Geriatr Psychiatry 18 (6):531-543.

doi:10.1097/JGP.0b013e3181cc0366

56. Mouzon DM, Taylor RJ, Keith VM, Nicklett EJ, Chatters LM (2017) Discrimination and psychiatric disorders among older African Americans. International journal of geriatric psychiatry $32(2): 175-182$

57. Seng JS, Lopez WD, Sperlich M, Hamama L, Meldrum CDR (2012) Marginalized identities, discrimination burden, and mental health: Empirical exploration of an interpersonal-level approach to modeling intersectionality. Social Science \& Medicine 75 $(12): 2437-2445$

58. Enticott JC, Meadows GN, Shawyer F, Inder B, Patten S (2016) Mental disorders and distress: Associations with demographics, remoteness and socioeconomic deprivation of area of residence across Australia. Australian \& New Zealand Journal of Psychiatry 50 (12):1169-1179

59. Dressel P, Minkler M, Yen I (1997) Gender, race, class, and aging: advances and opportunities. International Journal of Health Services 27 (4):579-600

60. Koehn S, Neysmith S, Kobayashi K, Khamisa H (2013) Revealing the shape of knowledge using an intersectionality lens: results of a scoping review on the health and health care of ethnocultural minority older adults. Ageing \& Society 33 (3):437-464 
61. Lane P, Tribe R, Hui R (2011) Intersectionality and the mental health of elderly Chinese women living in the UK. International Journal of Migration, Health and Social Care 6 $(4): 34-41$

62. Batten G (2019) Normalising mental illness for older adults. AIFS. https://aifs.gov.au/cfca/2019/02/13/normalising-mental-illness-older-adults-barrier-care. 2 October 2019

63. Wuthrich VM, Frei J (2015) Barriers to treatment for older adults seeking psychological therapy. International Psychogeriatrics 27 (7):1227-1236

64. McKay RC, J. Stevenson, J. McGowan, (2015) Psychiatry services for older people. A report on current issues and evidence to inform the development of services and the revision of RANZCP Position Statement 22. RANZCP. 2 October 2019

65. Temple JB, Kelaher M, Paradies Y (2019) Prevalence and context of racism experienced by older Aboriginal and Torres Strait Islanders. Australasian journal on ageing $38(1): 39-46$

66. Temple JB, Kelaher M, Paradies Y (2019) Experiences of Racism among Older Aboriginal and Torres Strait Islander People: Prevalence, Sources, and Association with Mental Health. Canadian Journal on Aging/La Revue canadienne du vieillissement:1-12

67. Enticott JC, Lin E, Shawyer F, Russell G, Inder B, Patten S, Meadows G (2018) Prevalence of psychological distress: How do Australia and Canada compare? The Australian and New Zealand journal of psychiatry 52 (3):227-238. doi: $10.1177 / 0004867417708612$

68. Isaacs A, Enticott J, Inder B, Meadows G (2018) Lower income levels in Australia are strongly associated with elevated psychological distress: Implications for healthcare and other policy areas. Frontiers in Psychiatry 9:536 


\section{University Library}

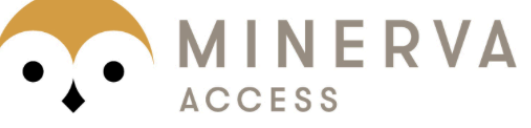

A gateway to Melbourne's research publications

Minerva Access is the Institutional Repository of The University of Melbourne

Author/s:

Temple, JB;Brijnath, B;Enticott, J;Utomo, A;Williams, R;Kelaher, M

Title:

Discrimination reported by older adults living with mental health conditions: types, contexts and association with healthcare barriers

Date:

2020-07-21

Citation:

Temple, J. B., Brijnath, B., Enticott, J., Utomo, A., Williams, R. \& Kelaher, M. (2020). Discrimination reported by older adults living with mental health conditions: types, contexts and association with healthcare barriers. SOCIAL PSYCHIATRY AND PSYCHIATRIC EPIDEMIOLOGY, 56 (6), pp.1003-1014. https://doi.org/10.1007/s00127-020-01914-9.

Persistent Link:

http://hdl.handle.net/11343/253847 